\title{
Estrategias de conformación y consolidación de semilleros de investigación en pregrado. Estudio de caso en una institución de educación superior en Colombia
}

Ninfa Vega-Monsalve

\section{RESUMEN}

Este artículo comprende las estrategias de conformación y consolidación utilizadas por dos semilleros de investigación de pregrado de una Institución de Educación Superior de Colombia, que ha generado procesos exitosos de formación para la investigación. El estudio fue de corte cualitativo, con enfoque hermenéutico y método de estudio de caso, se utilizaron entrevistas, observación participante, grupo focal y revisión del archivo documental del proceso. Se encontró que el éxito del proceso reside en la ejecución de cuatro momentos. La motivación, dinámica de trabajo, desempeño del docente líder y apoyo institucional. Los hallazgos comprenden algunas estrategias que podrían ser implementadas en otros escenarios.

Palabras clave: investigación educativa, grupos de investigación, grupos de estudio, Colombia.

Ninfa Vega-Monsalve

ninfavegamonsalve@gmail.com Colombiana. Magister en Gerencia del Talento Humano, Universidad de Manizales, Colombia. Directora de la Escuela de Gestión Estratégica (EGE), Colombia. Temas de investigación: gestión humana, seguridad y salud en el trabajo, educación y gestión del conocimiento. 


\section{Estratégias de conformação e consolidação de seminários de pesquisa em graduação. Estudo de caso em uma instituição de educação superior na Colômbia}

\section{RESUMO}

Este artigo compreende as estratégias de conformação e consolidação utilizadas por dois seminários de pesquisa de graduação de uma instituição de educação superior da Colômbia, que tem gerado processos exitosos de formação para a pesquisa. O estudo foi de corte qualitativo, com enfoque hermenêutico e método de estudo de caso, se utilizaram entrevistas, observação de participante, grupo focal e revisão do arquivo documental do processo. Se encontrou que o êxito do processo reside na execução de quatro momentos: motivação, dinâmica de trabalho, desempenho do docente líder e apoio institucional. As descobertas compreendem algumas estratégias que poderiam ser implementadas em outros cenários.

Palavras chave: pesquisa educativa, grupos de pesquisa, grupos de estudo, Colômbia.

\section{Strategies for the formation and consolidation of undergraduate research seedbeds. A case study in an institution of higher education in Colombia}

\section{ABSTRACT}

This article offers information about the conformation and consolidation strategies used by two undergraduate research seedbeds of an institution of higher education in Colombia, which has generated successful training processes for research. The study was qualitative, with a hermeneutic approach and a case study method, using interviews, participant observation, focus group and review of the documentary archive of the process. The study allowed to observe that the success of the process resides in the execution of four moments: motivation, work dynamics, performance of the leading teacher and institutional support. The findings include some strategies that could be implemented in other settings.

Key words: educational research, research groups, study groups, Colombia.

Recepción: 16/08/16. Aprobación: 18/05/18. 


\section{Introducción}

Este artículo forma parte del proyecto de sistematización de la experiencia de dos semilleros de investigación del pregrado de administración en salud ocupacional de una institución de educación superior (IES) con sede en el municipio de Bello, Colombia.

Tiene como propósito comprender las estrategias que han ayudado a la conformación y consolidación de estos dos casos, al igual que los retos que se les plantean en el futuro; en el entendido de que pueden servir como referencia a otros y con ello contribuir a la obtención de aprendizajes significativos y motivación de los estudiantes por los procesos de indagación, pensamiento crítico, innovación y reflexión de las ciencias. Estos semilleros de investigación formativa (SI) se han destacado a nivel institucional; su líder fue reconocido a través del premio a la excelencia investigativa 2014, a nivel regional y nacional, y los integrantes han participado como ponentes en encuentros organizados por la Red Colombiana de Semilleros de Investigación (Redcolsi), e incluso seleccionados como proyecto nacional en 2014.

\section{Marco teórico}

La ley 30 de 1992, por la cual se organiza el servicio público de la educación superior en Colombia, ha definido que una función sustantiva de las IES es la investigación, a partir de la cual se transforma y apropia el conocimiento y con ello se logra un nuevo concepto de competencia laboral (Vega Monsalve, 2016), en donde no sólo se generan conocimientos técnicos, sino se aporta para la construcción de un mejor tejido social. La Misión de sabios $(1996)^{1}$ indicó que el futuro del país dependía de los avances en esta materia y por ello para el siglo XXI se debía contar con al menos un investigador por cada mil habitantes. Reto que aún no se materializa, pues con una población de 45 millones sólo se cuenta con 7000 profesionales acreditados (OCyT, 2016), número muy inferior respecto a otros países como México, que cuenta con 435922 o Argentina que tiene 50785 investigadores registrados.

El no contar con una cantidad mayor de investigadores resta oportunidades para que el país desarrolle su potencial en innovación, creación de nuevos servicios y productos, mejora tecnológica de los sectores económicos que hoy día tiene y, en definitiva, progreso en la calidad de vida y desarrollo de sus ciudadanos.

Según el Observatorio Colombiano de Ciencia y Tecnología (OCyT, 2016), el 70\% de las investigaciones proviene de las universidades, por lo que fortalecer este campo es de vital importancia para el país. Una de las estrategias para mantener viva la cultura científica en estas instituciones es el desarrollo de procesos de formación en investigación, mediante la cual se forma a nuevos investigadores que comprendan la sistematicidad y rigurosidad del método científico, en la mayoría de los casos esto se hace a partir de procesos pedagógicos de aprender-haciendo, poco a poco se familiariza a los estudiantes con los conceptos y procedimientos propios de la ciencia hasta desarrollar en ellos capacidades de investigación (CNA, 2014; Valencia, Macías y Valencia, 2015).

En las IES la formación en investigación se ofrece a través de distintas opciones: monitorías investigativas, proyectos de grado, eventos académicos, pasantías investigativas, cursos dentro del currículo o participación en los SI. Este artículo trata sobre estos últimos. Definidos como grupos extracurriculares de origen espontáneo (García, 2009), donde no media pago por pertenecer (Chávez, Rodríguez, Lozano, Vargas y Lozano, 2015), de naturaleza autónoma e integrados por estudiantes motivados (Macea-Acuña, 2008; Quispe, Horta, Medina y

\footnotetext{
${ }^{1}$ En 1996 un grupo de reconocidos intelectuales colombianos, conformaron una comisión en la que analizaron la situación social del país y presentaron al Gobierno Nacional una propuesta que buscaba transformar la educación y propulsar el desarrollo. A dicha comisión se le llamó Misión de sabios.
} 
León, 2015), liderados por un docente con experiencia investigativa (Etzkowitz, 1993), donde se fortalece el aprendizaje y se contribuye a la consolidación de una cultura científica (Miyahira, 2009; Benjumea y Sánchez, 2011; González-Velázquez, 2014).

Como se nota en esta definición, el objetivo principal que gobierna la constitución de los semilleros de investigación es la de generar cultura investigativa en los estudiantes y para ello se requiere su voluntad y motivación; también, que estén liderados por docentes con acercamiento práctico al mundo investigativo, capaces de contagiar su pasión a los integrantes de estos grupos. Los SI en Colombia son una propuesta reciente - surgen a finales de los noventa- que poco a poco ha ido ganando espacios, al punto de convertirse en un gran movimiento académico que hoy reúne a 7500 SI de 366 IES (Redcolsi, 2016).

Varios estudios han registrado el aporte de estos grupos a la generación de cultura investigativa, transferencia del conocimiento y fortalecimiento del perfil profesional de los futuros graduados (OlmosPeñuela, Castro-Martínez y D'Este, 2014; Hurtado, Baños y Silvente, 2015). La formación en investigación que se desarrolla a través de los SI es una actividad pedagógica que transversaliza todas las asignaturas o cursos de la formación profesional pues permite estimular las habilidades de indagación, reflexión y análisis en los estudiantes; de igual forma, se han registrado fortalezas en el campo del desarrollo de competencias argumentativas (MaytaTristán, Cartagena-Klein, Pereyra-Elías, Portillo y Rodríguez-Morales, 2013). Los SI representan una alternativa eficaz que permite la mejora de la calidad del futuro profesional (Valencia et al., 2015).

Varios estudios por separado y en países distintos a Colombia han expuesto algunos factores que inciden en el éxito de los grupos de investigación, los factores que motivan a los estudiantes a permanecer en estos grupos no escapa a la teoría de grupos y a lo descrito en algunas investigaciones como las que se muestran a continuación:
- Compromiso de los estudiantes con el proceso, el cual se manifiesta de muchas formas: llegar puntuales a los encuentros, cumplir con los compromisos, estar presentes cuando se les convoca (Reyes, 2013), participar de forma activa de las discusiones y aportar nuevas ideas al grupo. El compromiso es la manifestación del grado de motivación del estudiante, la cual puede ser influenciada por estímulos del ambiente externo, su habilidad cognitiva y propia voluntad (García y Doménech, 2007).

- Liderazgo del docente, quien puede ser facilitador u obstaculizador del aprendizaje. Los estudiantes tienen la expectativa de que su tutor genere un ambiente agradable y justo, domine los conocimientos y los apoye (Reyes, 2013). Por esto se requiere un docente que entienda que el centro del proceso de enseñanza-aprendizaje no es él, sino los estudiantes. Su papel es acompañarlos y guiarlos por los distintos caminos de hacer ciencia, el que toma las decisiones y lidera su propio proyecto es el estudiante, los retos que se les plantean deben estar acordes con los diferentes modos de afrontar las tareas académicas y patrones de motivación y aprendizaje (García et al., 2007), buscando que cada estudiante se sienta capaz de ejecutar las tareas y llegar al logro esperado.

- La dinámica del trabajo parte de las estrategias que el docente líder sea capaz de generar para mantener la atención de los integrantes del SI, como lo plantea Lima (2014), es una labor permanente que se apoya en recursos intrínsecos y extrínsecos que tiene como propósito, además de asegurar el aprendizaje, la activa participación de los estudiantes.

- Apoyo institucional (Triadó y Aparicio, 2006; Altopedi, Hernández-De-La-Torre y López Yáñez, 2015), referido a facilitar los recursos económicos, humanos y de infraestructura para conformar y consolidar los procesos con 
los grupos. En este sentido, también se espera que haya acompañamiento a los docentes líderes, motivándolos para que implementen tutorías a partir de estrategias pedagógicas constructivas y experienciales.

Como se observa, estos factores refieren que la gestión exitosa depende del compromiso de todos los actores involucrados: institución, estudiantes y docentes. Los SI aportan de forma significativa a la cultura investigativa por lo que preocuparse por su pleno potencial es fundamental. A pesar de esto, al menos en el país, se presentan obstáculos para el desarrollo integral de estos grupos, por ejemplo, falta una política más decidida para su fomento y consolidación (Semana, 2016) y hay escases de docentes formados y motivados para asumir la dirección de estos grupos (Benjumea et al., 2011), sin mencionar que son escasos los estudios sobre el tema, de allí la relevancia de esta investigación, que comprende las estrategias de conformación y consolidación de semilleros de investigación en pregrado, a partir de la experiencia de dos casos, que aporta puntos de referencia para otros.

\section{Métodos}

Este estudio es de corte cualitativo, con enfoque hermenéutico buscó la comprensión y entendimiento de los sentidos de los discursos de los sujetos. En palabras de Echeverría (1997), el entendimiento a partir del diálogo y el ejercicio interpretativo. Se utilizó el método de estudio de caso, lo que permitió conocer en profundidad los dos casos estudiados, arrojando una descripción rica del fenómeno y generando nuevo y valioso conocimiento que puede enriquecer las prácticas de los semilleros de formación en investigación de pregrado.

Para asegurar el rigor y la credibilidad del estudio se cumplieron varios de los ítems contenidos en la lista de comprobación para la evaluación crítica de investigaciones cualitativas (COREQ) (Tong,
Sainsbury y Craig, 2007) que, aunque fue adoptada por revistas de salud, presenta buenas prácticas que vale la pena copiar. Se siguieron las recomendaciones del análisis y procesamiento de los datos, en el sentido de hacer explícitas las categorías preconcebidas con que se realizó la codificación de los datos, obtener retroalimentación de los participantes sobre los resultados de la investigación antes de la publicación de este artículo, proporcionar citas de apoyo de lo manifestado por los sujetos y emplear software para el almacenamiento, la búsqueda y la codificación y análisis de los datos.

La unidad de análisis correspondió a los SI del programa de pregrado Administración en Salud Ocupacional de la Facultad de Educación de una IES de Colombia, con presencia en varias regiones del país, más de 78000 estudiantes y reconocida por su cobertura a comunidades que no han tenido acceso a la educación superior por diversos factores, entre ellos el económico, llegando a que el 98\% de sus estudiantes pertenezcan a los estratos socioeconómicos más bajos (1, 2 y 3). El criterio de inclusión fue semilleros con una trayectoria superior a un año y reconocidos a nivel institucional por su consolidación y resultados (publicaciones, ponencias y organización de eventos).

Como mecanismo de validez externa, se empleó la lógica de la replicación (Yacuzzi, 2005), consistente en la selección de varios casos que guardan características similares: semilleros de investigación con dos y cuatro años de trayectoria, entre 7 y 9 integrantes, todos estudiantes de Salud Ocupacional de los últimos semestres, entre los 21 y 44 años. Se contó con un graduado del programa que en su momento fue fundador de uno de los semilleros y decidió quedarse luego de su graduación. Ninguno contaba con formación o experiencia previa en investigación. Casi el total de los grupos estaba integrado por mujeres. Todos contaban con al menos dos años de experiencia profesional en el área de salud ocupacional. 


\section{Consideraciones éticas}

Este estudio cumplió las consideraciones éticas consignadas en la Resolución 8430 de 1993 de Colombia que define este tipo de investigaciones como $\sin$ riesgo. Está acorde además con los planteamientos de Emanuel (1999) que, aunque se refieren a estudios clínicos, presenta recomendaciones que, en opinión del autor, todas las investigaciones deberían cumplir. De ahí el énfasis en el desarrollo de un estudio con valor social y validez científica, conveniente y transparente con la muestra.

\section{Diseño de instrumentos}

Se creó un protocolo de recogida de datos, se privilegió la interacción con los actores. Para aumentar la fiabilidad y confianza de la interpretación de los hallazgos, se utilizó la triangulación metodológica, consistente en usar distintos instrumentos que confirmaran los resultados, así, se aplicaron 16 entrevistas presenciales a profundidad con los estudiantes y profesor líder de semilleros, también con directivas de la IES. Dichos encuentros duraron entre 45 y 80 minutos, realizados en dos fechas distintas. También dos grupos focales con los integrantes de los grupos, observación participante de cuatro reuniones del grupo. Revisión del archivo documental del proceso de conformación, proyectos y eventos académicos ejecutados, memorias de ponencias e informes de seguimiento de los grupos.

La triangulación metodológica aportó validación de los hallazgos, pues redujo el sesgo propio de los instrumentos cualitativos; a pesar de usar distintos instrumentos los participantes mostraron consistencia en los factores de éxito que consideran ayuda a la conformación y consolidación de los SI; el uso posterior a las entrevistas de grupos focales permitió profundizar en los aspectos novedosos que se obtuvieron de los participantes.

Las categorías teóricas se obtuvieron del análisis de los discursos de los participantes, según los cuales existe una lista de factores que inciden en su motivación para pertenecer a los SI, y unas estrategias que han permitido la consolidación de estos grupos. Luego estos factores fueron ampliados y fundamentados con referenciación teórica, buscando estudios que los validaran o confrontaran; al final se construyó una lista de factores (ver tabla 1) a partir de la cual se diseñaron los instrumentos del proyecto.

Para garantizar la consistencia y fiabilidad del estudio, los instrumentos fueron construidos a partir de la comprensión de las categorías y sometidos a juicio de expertos - revisaron que el contenido de las preguntas diera cuenta de las categorías estudiadas y el lenguaje y estilo de redacción fuera comprensible - y prueba piloto.

\section{Registro y análisis de los datos}

Los registros se hicieron a detalle para recuperar la información verbal y no verbal de los actores. Las entrevistas y grupos focales fueron grabados y luego transcritos fielmente, más tarde procesados con el software Atlas Ti, adoptado por esta investigación por su aporte en el método comparativo constante, que consiste en comparar una y otra vez, de manera sistemática, los códigos nuevos obtenidos (San Martín Cantero, 2014). Se utilizó la técnica de análisis del discurso, identificación y clasificación estructural de dimensiones estratégicas y búsqueda de patrón de comportamiento común. Se realizaron matrices de categorías que permitieron realizar análisis cruzados de evidencias.

\section{Hallazgos Estrategias de conformación}

Se encontró que el éxito del proceso de conformación de los SI reside en la ejecución de seis momentos, los cuales se unen metódicamente y no sólo garantizan la conformación de los grupos sino la voluntad política de la IES para promover su funcionamiento. Cada momento responde al anterior y reporta al siguiente (ver figura 1). 
Tabla 1. Categorización para el trabajo de campo y análisis de resultados

\begin{tabular}{|c|c|c|c|c|}
\hline Categorías & Subcategoría & Descriptores & Atributos & Ítem \\
\hline \multirow{6}{*}{$\begin{array}{l}\text { Factores de } \\
\text { motivación para } \\
\text { pertenecer a } \\
\text { semilleros de } \\
\text { investigación }\end{array}$} & \multirow{3}{*}{ Intrínsecos } & Disciplina & $\begin{array}{l}\text { Disciplina y responsabilidad } \\
\text { para cumplir con los } \\
\text { compromisos del semillero. }\end{array}$ & Revisión de compromisos. \\
\hline & & $\begin{array}{l}\text { Motivación para el } \\
\text { aprendizaje }\end{array}$ & $\begin{array}{l}\text { Desear conocer y aprender } \\
\text { más. }\end{array}$ & Proyecto de vida personal. \\
\hline & & Pertenencia & Sentimiento de hacer parte de. & $\begin{array}{l}\text { Orgullo personal por } \\
\text { pertenecer. }\end{array}$ \\
\hline & \multirow{3}{*}{ Extrínsecos } & Acompañamiento docente & $\begin{array}{l}\text { Motivación permanente del } \\
\text { docente. }\end{array}$ & Reuniones permanentes. \\
\hline & & Ruta de trabajo & $\begin{array}{l}\text { Plan de trabajo con metas e } \\
\text { indicadores. }\end{array}$ & Cronograma de actividades. \\
\hline & & $\begin{array}{l}\text { Retos y resultados } \\
\text { tangibles }\end{array}$ & $\begin{array}{l}\text { Ver que los esfuerzos valen la } \\
\text { pena porque dan resultados. }\end{array}$ & $\begin{array}{l}\text { Actas de reunión, } \\
\text { seguimientos. }\end{array}$ \\
\hline \multirow{5}{*}{$\begin{array}{l}\text { Estrategias de } \\
\text { conformación y } \\
\text { consolidación }\end{array}$} & \multirow{2}{*}{ Del proceso } & $\begin{array}{l}\text { Seguimiento y } \\
\text { acompañamiento }\end{array}$ & $\begin{array}{l}\text { Revisión de compromisos y } \\
\text { retroalimentación. }\end{array}$ & Comunicación permanente. \\
\hline & & Planificación del trabajo & $\begin{array}{l}\text { Plan de trabajo con metas e } \\
\text { indicadores. }\end{array}$ & Cronograma de actividades. \\
\hline & $\begin{array}{l}\text { Por parte del } \\
\text { docente líder }\end{array}$ & $\begin{array}{l}\text { Motivación y compromiso } \\
\text { del docente líder }\end{array}$ & $\begin{array}{l}\text { Motivación permanente del } \\
\text { docente líder. }\end{array}$ & $\begin{array}{l}\text { Reuniones y comunicación } \\
\text { permanente. }\end{array}$ \\
\hline & \multirow[t]{2}{*}{$\begin{array}{l}\text { Por parte de } \\
\text { la IES }\end{array}$} & Disponibilidad de recursos & $\begin{array}{l}\text { Prestar los recursos para el } \\
\text { desarrollo de las acciones } \\
\text { (tiempo docente, financiación } \\
\text { de investigaciones). }\end{array}$ & $\begin{array}{l}\text { Componente formación en } \\
\text { investigación. }\end{array}$ \\
\hline & & Formación en investigación & $\begin{array}{l}\text { Articulación de los procesos } \\
\text { de formación en el aula. }\end{array}$ & $\begin{array}{l}\text { Proyectos educativos } \\
\text { integrados. }\end{array}$ \\
\hline
\end{tabular}

\section{Figura 1. Estrategias de conformación de los semilleros de investigación estudiados}

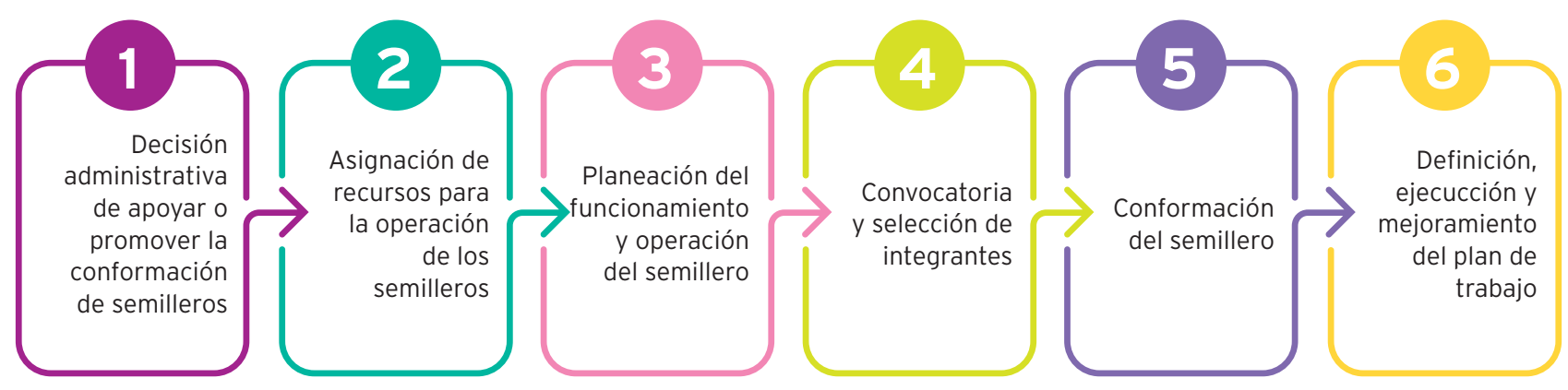


El SI más antiguo nació en respuesta a la solicitud de la dirección de investigaciones de la institución que estaba deseosa de más grupos; el segundo se genera por motivación personal del docente líder, que era abordado por estudiantes que querían pertenecer a un semillero. No se encontró que la naturaleza de la conformación incidiera en la dinámica de los grupos, pero sí que los resultados positivos del primero sirvieron como motivación para que otros estudiantes desearan hacer parte del segundo.

En ninguno de los casos existen pruebas de preselección para los integrantes o pago económico para participar, aunque realizan actividades de recolección de fondos que son controlados por un integrante nombrado de manera democrática como tesorero, los recursos son usados para financiar algunos gastos: papelería, refrigerios, viajes, contratación de servicios de diseño gráfico, entre otros.

En una alta proporción la motivación inicial de los estudiantes fue cumplir con un requisito para optar grados, luego de un tiempo el abanico de razones se amplío: les gustó investigar, el semillero les fortaleció personalmente, sus competencias educativas aumentaron y la dinámica del grupo les gustó. Varios han participado como ponentes en eventos académicos y todos adelantan un proyecto de investigación, lo que se vuelve en una motivación más para continuar en los grupos.

El proceso de conformación requirió apoyo y asignación de recursos por parte de la IES, referidos en este caso a la asignación de horas en el plan de trabajo del docente para que acompañará la operación de los grupos. Los entrevistados hicieron énfasis en que, a pesar de iniciar sin recursos, con el tiempo el alcance del SI creció y con ello la necesidad de contar con apoyo para la ejecución del plan de trabajo. Señalan como relevante que antes de arrancar la idea de crear un semillero, se cuente con el visto bueno de la IES, la que puede convertirse en facilitadora u obstaculizadora de su progreso.

Dentro de los momentos, se evidenció un marcado interés por las actividades de planificación, es así como los momentos tres y seis responden a esta lógica. Los distintos actores coincidieron en señalar que, aunque los SI son grupos espontáneos con interés por la formación y no por la producción académica, deben contar con una ruta de trabajo que oriente los encuentros y plantee retos e indicadores de evaluación del progreso de los grupos. Revelaron que, de no existir una ruta clara de trabajo, se corre el riesgo de perderse en detalles y no avanzar en el reconocimiento de los pasos del método científico.

En el momento cuatro, referente a la convocatoria de los estudiantes, en ambos semilleros primó la comunicación directa con los interesados, se les convocó a través de correos electrónicos, invitaciones en página web, exposición de la experiencia en eventos organizados por la misma IES, afiches en lugares estratégicos como pasillos muy transitados y cafeterías, e información en las aulas de clase.

Se encontró que las siguientes acciones pueden favorecer la convocatoria de nuevos integrantes:

- Ser claros con los estudiantes, indicando los alcances del proyecto, tiempo que le implicará su participación, beneficios que se perciben al participar.

- Citarlos a una reunión grupal en donde se amplié información sobre el proceso y se respondan todas sus inquietudes.

- Crear algún tipo de filtro para pertenecer al semillero, se recomienda privilegiar el ingreso de estudiantes de semestres iniciales, ya que cuando el semillero se conforma sólo con estudiantes de últimos semestres, se corre el riesgo de que, al graduarse, se retiren y por tanto el semillero desaparezca.

- Aprovechar los cursos de introducción a la investigación para presentarles informes finales de investigación realizadas por estudiantes de semilleros y contarles de los logros de los semilleros del programa, de esa forma, cuando haya las convocatorias, tendrán mayor interés por el tema. 
- Asegurar que la información de la convocatoria sí les llegue, por tanto, hacerlo con suficiente anticipación y a través de varios canales. Uno puede ser entregar la publicidad a los colegas profesores, para que en sus clases la distribuyan entre los estudiantes.

- A lo largo del semestre ir creando un banco de postulaciones, con los estudiantes que van mostrando su interés por formar parte de los semilleros, de esa manera, cuando se requieran estudiantes se puede contactar de forma directa a quienes ya habían expresado interés.

\section{Estrategias de consolidación}

Esta respondió a cuatro momentos: motivación de los estudiantes, dinámica de trabajo, desempeño del docente líder y apoyo institucional, que se reúnen en dos tipos de motivación: interna y externa (ver figura 2).
En cuanto a la motivación interna, que es de dominio personal, se ubica la disciplina y el grado de interés por pertenecer al grupo. Esto último se desarrolla según los consultados cuando se encuentra sentido a lo que se hace dentro del SI. Uno de ellos señaló: "el mejor enganche [motivación] va a ser si la investigación que se realiza siempre está relacionada con lo que el estudiante está estudiando o investigaciones afines que le puedan complementar la profesión que uno ya tiene" (E5, comunicación personal, 9 de mayo de 2015).

El segundo factor, llamado motivación externa, corresponde con el apoyo que da la institución, el compromiso y acompañamiento del docente líder y los sentimientos y clima que se lograron desarrollar en la dinámica de los grupos. Los entrevistados dan un profundo interés a los factores del proceso y la institución en estos se destacan cuatro aspectos:

\section{Figura 2. Estrategias de consolidación de los semilleros de investigación estudiados}

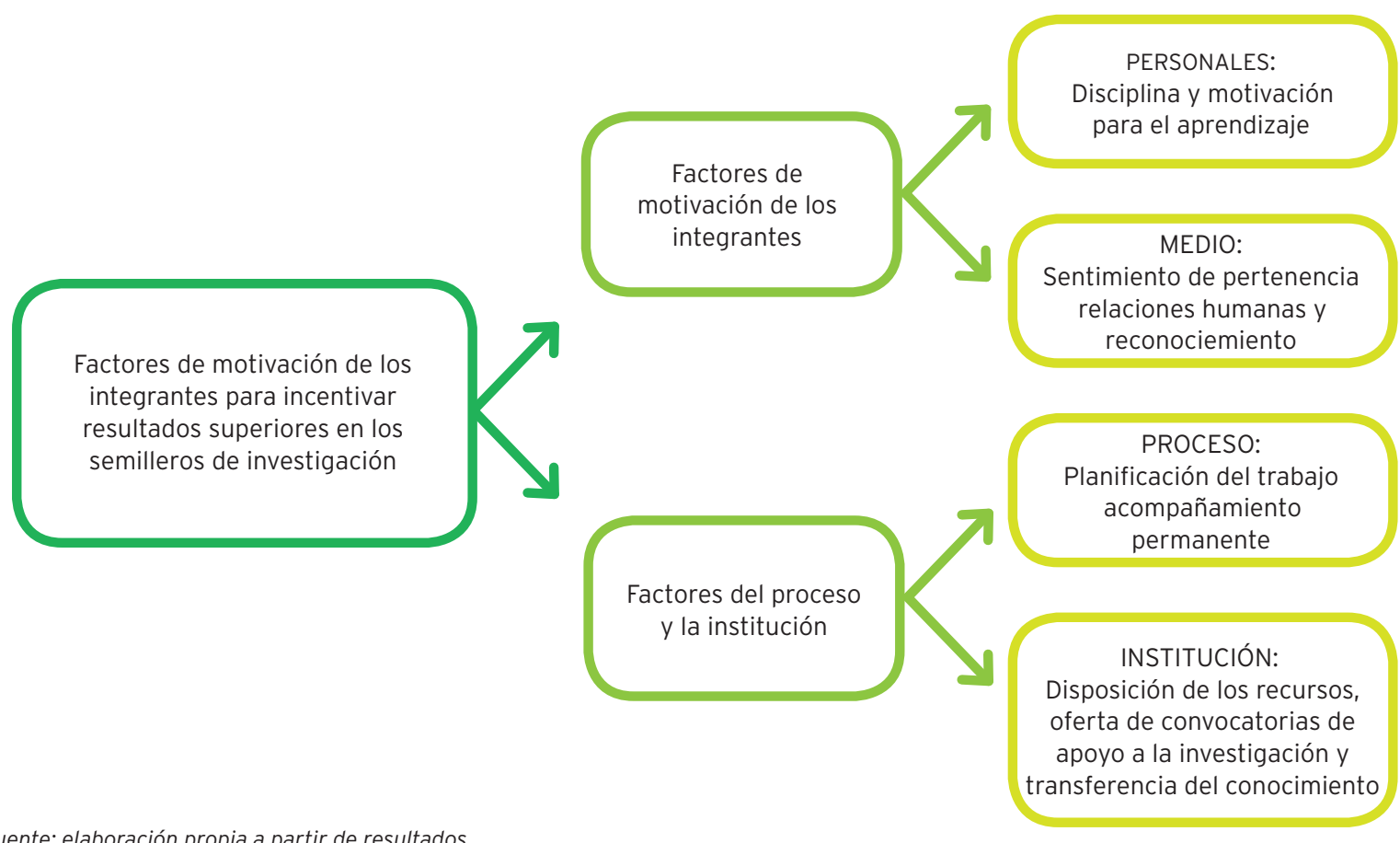


- Seguimiento y acompañamiento permanente. Los estudiantes refieren que necesitan que el docente permanezca conectado con el proceso, haciendo revisión y retroalimentación en tiempo real, que no sólo haya dedicación en los encuentros presenciales sino a través de los medios de comunicación virtuales. El recordar las tareas, verificar la calidad de las entregas que los estudiantes hacen es clave en el proceso. Algunas actuaciones del docente han ayudado a incrementar la motivación de los estudiantes, por ejemplo: seleccionar aquellas tareas que ofrezcan retos y desafíos razonables por su novedad, variedad o diversidad, ayudarlos en la toma de decisiones, fomentar su responsabilidad e independencia y desarrollar habilidades de autocontrol. Centrarse sobre el progreso y mejora individual, reconocer el esfuerzo y trasmitir la visión de que los errores son parte del proceso. Estrategias que pueden facilitar la permanencia de los estudiantes aun después de su graduación, a pesar de que ello les demande tiempo y esfuerzo que no será visible o rápidamente recompensado.

- Metodología de acción. Según los entrevistados les ha funcionado que el trabajo sea bajo la metodología de educación experiencial en donde juntos y paso a paso se va aprendiendo a investigar, alguien manifestó "la mejor manera para que los estudiantes apropien los pasos del método científico es cuando lo viven, eso ayuda a reducir la ansiedad propia de aprender algo nuevo" (E7, comunicación personal, 16 de abril de 2015).

- Compromiso por parte del líder del proceso. Los procesos de liderazgo de ambos semilleros presentan un factor clave y es la motivación y compromiso del docente con el proceso. Los estudiantes refieren que sienten que la labor del docente no es sólo por estar asignada en su plan de trabajo, sino que existe pasión por lo que se realiza y compromiso personal con el grupo. Pasión que les ha servido a ellos de motivación para pertenecer y mantenerse en el SI.

- Estímulos para los integrantes de los Semilleros. Aunque los entrevistados lo refieren como un reto aún no logrado, indican que es importante que la institución promueva espacios de "privilegio" y reconocimiento para los estudiantes que hacen parte de los semilleros. Algunos estímulos que proponen son: descuentos en matrículas, becas de estudio, bonificaciones económicas, entre otras.

En cuanto a los retos o situaciones problemáticas que presentan, ambos casos coincidieron en los siguientes elementos:

- Horas en el plan de trabajo del docente para que acompañe los grupos

- Participación en eventos externos

- Trabajo en red con otros semilleros

- Vinculación de estudiantes de otras disciplinas

- Recursos para ejecución de proyectos de investigación

- Articulación de los semilleros con los espacios de formación investigativa contemplados en el currículo

- Estímulos para quienes integran los semilleros.

\section{Discusión}

Esta investigación pretendió comprender las estrategias de conformación y consolidación utilizadas por dos SI de pregrado de una IES de Colombia y encontró que el éxito del proceso reside en la ejecución de cuatro momentos. La motivación, dinámica de trabajo, desempeño del docente líder y apoyo institucional.

Los resultados de este estudio son similares a otros (Triadó et al., 2006) en los que se señala la importancia de contar con recursos para ofrecer estímulos a los integrantes que favorezcan su continuidad a pesar de haber egresado de la institución. El número 
de integrantes de los dos casos coincide con lo recomendado (Altopiedi et al., 2015) en el sentido de que un grupo numeroso impide el seguimiento y acompañamiento casi personalizado, que es lo requerido en este tipo de experiencias. Aunque difiere de lo encontrado por Horta y Lacy (2011) quienes mostraron que el tamaño total del grupo guarda escasa relación con la productividad en su conjunto.

En cuanto al poder de convocatoria de los semilleros, se coincide con García (2009) en cuanto a que, a pesar de no existir generosos incentivos institucionales, estos grupos logran seguir activos y producir por encima de lo requerido. Sin embargo, llama la atención el fenómeno de deserción o retiro de estudiantes al momento de graduarse, a pesar de que manifiestan estar muy satisfechos e interesados con el semillero, ello podría darse por la falta de estímulos para que continúen y por la condición de estudiantes/trabajadores, lo que justifica que, al momento de graduarse, prefieran dedicarse de forma exclusiva a lo que les genera ingresos: su empleo.

En cuanto a la relevancia manifestada por los entrevistados de sostener relaciones cercanas y un ambiente de familiaridad, se concuerda con lo expuesto por Hoffman et al. (2013), que refieren la necesidad de comprender estas dinámicas sociales y más cuando el proceso investigativo exige un alto nivel de disciplina y compromiso (Quispe et al., 2015).

$\mathrm{Al}$ igual que en los estudios de Etzkowitz (1993), en los casos estudiados los estudiantes se agrupan alrededor de un docente con experiencia investigativa. Se comparte lo ya descrito por Altopiedi et al. (2015), en donde el foco es la tarea académica. Tal y como lo indican Chávez et al. (2015), al ser la participación voluntaria y sin pago, incrementa las posibilidades de los estudiantes por participar. Por último se coincide con Miyahira (2009) y Reyes (2013) en cuanto a que el acompañamiento de los semilleros requiere un perfil distinto de docente.

Por el contrario, los hallazgos contradicen los resultados de otros estudios como por ejemplo el de
Altopiedi et al. (2015) en donde por la disparidad de edad, los integrantes mayores ven como hijos a los más jóvenes. En estos casos la relación es entre iguales. Una razón puede ser las diferencias culturales de las poblaciones y que en el caso de la primera investigación los mayores ya contaban con experiencia investigativa.

También se encuentran diferencias respecto a la poca autonomía de los estudiantes para seleccionar el objetivo de investigación, en estos casos se parte del interés personal de los integrantes, quienes decidieron qué estudios realizar sobre los temas de su interés. Distinto a la experiencia de Sierra, Rojas y López (2009), en los casos analizados no existen pruebas de selección para nuevos integrantes, los filtros se activan durante el proceso cuando son retirados por incumplimiento de alguna de las reglas acordadas de forma previa. Tampoco hay presencia de estudiantes de otras disciplinas, como en el estudio de Rivera, Osorio, Tangarife y Arroyave (2000).

Este estudio genera importantes contribuciones. En el sentido académico abre nuevos interrogantes que se recomienda indagar. Por ejemplo: ¿cuál es la verdadera diferencia y naturaleza de los SI con los grupos de investigación, cuando en el contexto institucional se les está formalizando y pidiendo resultados?, ¿qué tan espontánea es la conformación de los SI cuando para su consolidación se requiere el visto bueno de las IES?, ¿cómo crear mecanismos de verificación y control que no obliguen a los SI a desconocer o relegar su naturaleza pedagógica, introductoria y formativa?, y ¿cómo los SI aportan a la generación de competencias laborales como las que describe Vega-Monsalve (2016).

También abre la reflexión frente a que las instituciones de educación superior deben contribuir a que el estudiante capte la complejidad de la realidad social, la red de problemas y causalidades, las relaciones e integralidad de los sucesos, y esto puede lograrse a través del componente investigativo que actúa como un despertador o una alarma frente a 
la comprensión holística de las realidades, la indagación y la crítica de lo que se le enseña y a la valoración de sus propias capacidades para encontrar la respuesta a los problemas que el mundo cotidiano le presenta.

A nivel práctico presenta estrategias que pueden ser transferidas a otros SI para fortalecer su constitución y consolidación.

\section{Conclusiones}

La realización de este estudio permite concluir que la constitución y consolidación de los SI es el resultado del engranaje coordinado entre la IES, los integrantes del grupo y el docente líder. A pesar de que estos grupos han sido creados como una estrategia de formación en investigación, requieren un especial cuidado de la dinámica y calidad de las relaciones humanas con las que se aborda el trabajo.

Las IES tienen varios retos en la consolidación de este tipo de estrategias:
- Crear formas de estímulo y retención de los estudiantes una vez se gradúan y egresan de la institución.

- Asignar horas en los planes de trabajo de los docentes que lideran los semilleros y aportar los recursos para su funcionamiento.

- Diseñar un perfil especial para los docentes que se encargarán de esta labor, reconociendo que no sólo es necesario que cuente con formación y experiencia investigativa, sino que también disfruten digiriendo estudiantes y transmitiendo sus conocimientos.

- Planear una forma de evaluación y seguimiento que no desconozca la naturaleza formativa y no productiva de los SI.

- Integrar esta experiencia con los cursos que dentro del currículo ofrecen formación investigativa. 


\section{Referencias}

Altopiedi, M., E. Hernández-de la-Torre y J. LópezYáñez, J. (2015), "Características relevantes de grupos de investigación destacados en Andalucía”, en Revista Iberoamericana de Educación Superior, 6 (16), pp. 126-142.

Benjumea, E., y M. Sánchez (2011), "La investigación formativa en una institución oficial de educación superior", en Teoría y Praxis Investigativa, Fundación Universitaria del Área Andina, vol. 6, pp. 11-16.

Chávez, K., J. Rodríguez de Ita, J. Lozano, G. Vargas y F. Lozano (2015), "Desarrollo e implementación de un curso de investigación para estudiantes de ciencias de la salud: una propuesta para estimular la producción científica" en Investigación en Educación Médica, 4 (15), pp. 161-169.

Consejo Nacional de Acreditación (GNA) (2014), "Lineamientos para la acreditación de programas de pregrado", Bogotá, CNA.

Echeverría, R. (1997), El búho de Minerva, Santiago, Dolmen.

Emanuel, E. (1999), “¿Qué hace que la investigación clínica sea ética? Siete requisitos básicos”, en A. Pellegrini Filho y R. Macklin (eds.), Investigación en sujetos humanos: experiencia Internacional, Organización Panamericana de la Salud/Organización Mundial de la Salud, Programa Regional de Bioética, División de Salud y Desarrollo Humano, Serie Publicaciones, pp. $43-44$.

Etzkowitz, H. (1993), "Individual investigators and their research groups", en Minerva, vol. 30, pp. 28-50.

García, C. (2009), "Orígenes y dinámica de los semilleros de investigación en Colombia”, en L. Molineros (ed.), Orígenes y dinámica de los semilleros de investigación en Colombia. La visión de los Fundadores, Popayán, Taller Editorial Universidad del Cauca, pp. 20-28.

García, F. y F. Doménech (2007), "Motivación, aprendizaje y rendimiento escolar", en Revista Electrónica de Motivación y Emoción, 1(0).

González-Velázquez, S. (2014), "La investigación y su aporte al conocimiento. La experiencia de Enfermería en la Facultad de Estudios Superiores Zaragoza de la UNAM", en Enfermería Universitaria, 11 (2): pp. 45-46.

Hoffman et al. (2013), "The methodological illumination of a blind spot: information and communication technology and international research team dynamics in a higher education research program", en Higher Education, disponible en <http://dx.doi.org/10.1007/ s10734-013-9692-y>.

Horta, H. y T. A. Lacy (2011), "How does size matter for science? Exploring the effects of research unit size on academics scientific productivity and information exchange behaviors", en Science and Public Policy, vol. 38, núm. 6, pp. 449-460.

Hurtado, M. J. R., R. V. Baños y V. B. Silvente (2015), "La investigación formativa como metodología de aprendizaje en la mejora de competencias transversales", en Procedia-Social and Behavioral Sciences, 196, pp. 177-182.

Ley No. 30 de 28 de diciembre de 1992 de la República de Colombia, por la cual se organiza el servicio público de la Educación Superior, disponible en <http://www. cna.gov.co/1741/articles-186370_ley_3092.pdf>.

Lima, E. R. (2014), "Cómo motivar a los alumnos y desarrollar la creatividad en el aula", en Revista de la educación en Extremadura, ISSN: 1989-9041.

Macea-Acuña, F. (2008), "Aprender investigación: investigando y administración", en Administración y Desarrollo, pp. 43-53.

Mayta-Tristán, P., R. Cartagena-Klein, R. PereyraElías, A. Portillo y A. J. Rodríguez-Morales (2013), "Latin American medical students' appraisal on university scientific research training" en Revista Médica de Chile, 141 (6), pp. 716-22.

Miyahira, J. M. (2009), "La investigación formativa y la formación para la investigación en el pregrado", en Rev Med Hered, 20 (3), pp. 119-122.

Observatorio Colombiano de Ciencia y Tecnología (OCyT) (2016), Indicadores de ciencia y tecnología, Colombia 2015, Santafé de Bogotá, Ántropos.

Olmos-Peñuela, J., E. Castro-Martínez y P. D'Este (2014), "Knowledge transfer activities in social sciences and humanities: explaining the interactions 
of research groups with non-academic agents", en Research Policy, 43(4), pp. 696-706.

Presidencia de la Republica de Colombia (1996), "Informe de la misión de sabios Colombia: al filo de la oportunidad misión ciencia, educación y desarrollo", núm. 1, Santafé de Bogotá, Tercer mundo editores.

Quispe, G. B., S. H. Horta, C. E. Medina y P. A. León (2015), “Apreciación sobre capacitación en investigación y publicación científica en estudiantes universitarios", en Investigación en Educación Médica, 4(13), pp. 50-51.

Redcolsi (2016), Seguimos creciendo, [Internet] disponible en <http://fundacionredcolsi.org/web/index.php/2uncategorised/92-about-us $>$.

Reyes, C. (2013), "Una experiencia en la enseñanza de la investigación educativa en el marco de "proyecto aula" de la Universidad Veracruzana", en Revista Mexicana de Investigación Educativa, pp. 735-768.

Rivera, B., O. Osorio, D. Tangarife y J. Arroyave (2000), "Los semilleros de formación de jóvenes investigadores: La experiencia de ASPA", en Revista Universidad de Caldas, 19(1), pp. 7-18.

San Martín Cantero, Daniel (2014), “Teoría fundamentada y Atlas.ti: recursos metodológicos para la investigación educativa", en Revista Electrónica de Investigación Educativa, 16(1), pp. 104-122.

Semana (2016) "Colombia, un mal país para ser investigador", disponible en <http://www.semana. com/educacion/articulo/estudio-de-la-red-de- indicadores-de-ciencia-y-tecnologia-asegura-quecolombia-tiene-7000-investigadores/461104>.

Sierra, Z., A. Rojas y G. López (2009), "El Semillero de Investigación... Un espacio de vida", en L. Molineros (editor), Orígenes y dinámica de los semilleros de investigación en Colombia. La visión de los Fundadores, Popayán, Taller Editorial Universidad del Cauca, pp. 58-88.

Tong, A., P. Sainsbury y J. Craig (2007), "Consolidated criteria for reporting qualitative research (COREQ): A 32-item checklist for interviews and focus groups", en International Fournal for Quality in Health Care, 19(6), pp. 349-357, DOI: http://doi.org/10.1093/intqhe/ mzm042.

Triadó, X. y P. Aparicio (2006), El perfil de los grupos de investigacion màs competititos. Estudio del caso de la Universidad de Barcelona, Barcelona, AEDEM.

Valencia, J., J. Macias y A. Valencia (2015), "Formative research in higher education: some reflections", en Procedia-Social and Behavioral Sciences, 176, pp. 940-945.

Vega Monsalve, N. (2016), "Neocompetencias, nuevo enfoque de competencias laborales en salud ocupacional", en Educación Médica Superior, 30(3), recuperado de <http://www.ems.sld.cu/index.php/ ems/article/view/802/428>.

Yacuzzi, E. (2005), El estudio de caso como metodología de investigación: teoría, mecanismos causales, validación, Universidad del CEMA, 1-38, <http://doi.org/ Article>.

Cómo citar este artículo:

Vega-Monsalve, Ninfa (2019), "Estrategias de conformación y consolidación de semilleros de investigación en pregrado. Estudio de caso en una institución de educación superior en Colombia", en Revista Iberoamericana de Educación Superior (RIES), México, UNAM-IISUE/Universia, vol. X, Núm. 27, pp. 216-229, DOI: dx.doi.org/10.22201/ iisue.20072872e.2019.27.344 [consulta: fecha de última consulta]. 